\title{
Shaping Planetary Nebulae by Jets
}

\author{
Muhammad Akashi \\ Department of Physics, Technion - Israel Institute of Technology \\ akashi@physics.technion.ac.il
}

\begin{abstract}
Summary. We conduct 2D axisymmetrical hydrodynamical simulations to investigate the interaction of a collimated fast wind (CFW; wide jets) with a spherical AGB wind. The code includes radiative cooling. We find that the shape of the planetary nebula $(\mathrm{PN})$ is sensitive to the exact mass loss history of the AGB wind, and the opening angle of the CFW. Some typical PN morphologies are obtained, but many other observed morphologies seem to require more ingredients than what we assume in our present simulations, e.g., equatorial AGB wind, and ionization and fast wind during the PN phase. The hot bipolar bubble formed by the jets is an X-ray source.
\end{abstract}

Key words: ISM: jets and outflows; planetary nebulae: general

\section{Numerical Simulations}

Our simulations were performed using Virginia Hydrodynamics-I (VH-1), a high resolution multidimensional astrophysical hydrodynamics code developed by John Blondin and co-workers (Blondin et al. 1990; Stevens et al., 1992; Blondin 1994). We have added radiative cooling to the code at all temperatures $T>T_{\min }$, where the gas temperature is bound from below at $T_{\min }=300-1000 \mathrm{~K}$. Radiative cooling is carefully treated near contact discontinuities, where large temperature gradients can lead to unphysical results.

We simulate axisymmetrical morphologies. This allows us to use axisymmetrical grid, and to simulate one quarter of the meridional plane. There are 208 grid points in the azimuthal $(\theta)$ direction of this one quarter and 208 grid points in the radial direction. The radial size of the grid points increases with radius. In these simulations the grid extends from $10^{15} \mathrm{~cm}$ to $4 \times 10^{17} \mathrm{~cm}$.

Before the jet is launched $(t=0)$, the grid is filled with slow wind having a speed $v_{1}=10 \mathrm{~km} \mathrm{~s}^{-1}$ and mass loss rate $\dot{M}_{1} \sim 10^{-5} M_{\odot} \mathrm{yr}^{-1}$, with small variations between the runs. We launch a collimated fast wind from the first 20 zones attached to the inner boundary of the grid. The jet is uniformly 
ejected within an angle (half opening angle) $\alpha(0 \leq \theta \leq \alpha)$. For numerical reasons a weak slow wind is injected in the sector $\alpha<\theta \leq 90^{\circ}(\theta=0$ along the symmetry axis - vertical in the figures here).

\section{Results and Summary}

We try to explain PN shaping and formation processes by simulating jets interacting with the AGB wind. The parameter space for these types of flows is huge. We run more than 200 simulations, in which we tried many values of the $\mathrm{CFW}$ (jets) mass loss rate, velocity, and opening angle.

Another parameter that was used is the mass loss rate history of the AGB wind. For example, in a model presented here (figure 2), we assume that some period $\Delta t$ before the beginning of the jet-launching phase the slow wind mass loss rate increased by some factor $k$; e.g., in the run presented in figure 2 $\Delta t=950 \mathrm{yr}$ and $k=6$. We found that the mass loss history of the AGB wind plays a significant role in the shaping process. In some runs the AGB wind initial temperature was set to $10,000 \mathrm{~K}$ instead of $1,000 \mathrm{~K}$, with a noticeable influences on the final shape of the PN (compare the two panels in figure 2). Taking the AGB wind to be at $\sim 10,000 \mathrm{~K}$ is relevant to system where the accreting companion is a WD. If the accretion rate is high enough the WD sustains a constant nuclear burning, and it is very hot and luminous, as super-soft X-ray sources (e.g., Starrfield et al. 2004). Such an accreting WD might maintain the entire AGB wind ionized.

We were able to reproduce some physical properties of PNs, but not all desirable properties. It is very difficult, and might be impossible, to get the entire range of properties with our limited numerical code. We must add more ingredients to the code. Examples are pulsed jets, precessing jets, dense equatorial outflow and ionization fronts at later times.

We presented results of four numerical runs that migh match real PNs. Our main results are as follows:

1. We show that different PNs morphologies could be strongly dependent on the AGB wind mass loss rate history.

2. We have an evident that the final morphology is very sensitive also to the assumed initial temperature. For $T_{i}=1000 \mathrm{~K}$ and $T_{i}=10000 \mathrm{~K}$ we get two different evolutions of the planetary nebula (figure 21).

3 . The dense finger along the symmetry axis (in figure 2 top) result from instabilities. In reality, it would be more extended and not so narrow. It is forced to the symmetry axis by the numerical code.

4. In one case we obtain low density finger (in figure 2) protruding to the upper right form a torus. However, it is also a result of an instability, and we expect that in real systems there will be several such fingers. In particular, if there is a departure from axisymmetry due to the orbital motion, we would expect the fingers to be similar but not identical in the two sides of the equatorial plane. 

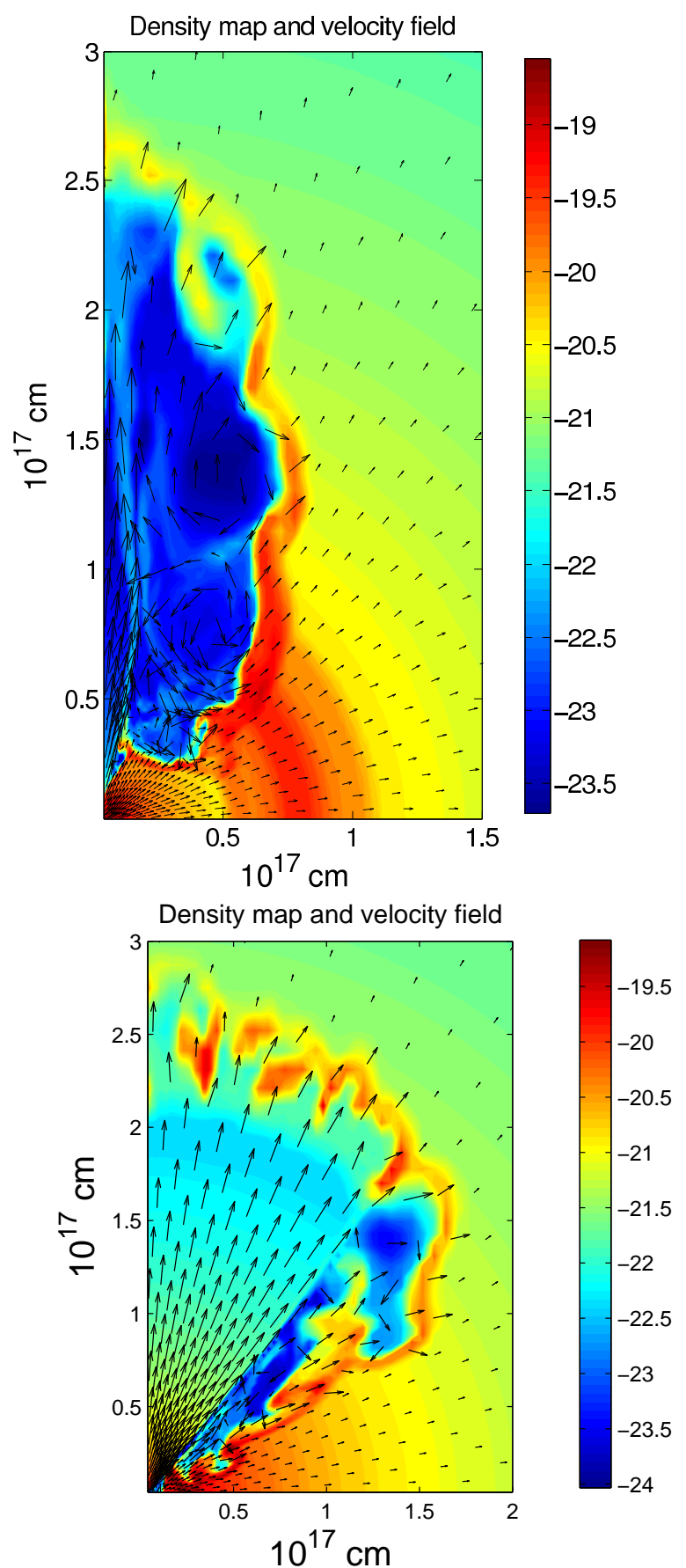

Fig. 1. Top: Density plot at $t=2000 \mathrm{yr}$ (after jet launching starts) for a case where the slow wind mass loss rate has increased by a factor of five to $\dot{M}_{1}=$ $5 \times 10^{-5} M_{\odot} \mathrm{yr}^{-1} 950 \mathrm{yr}$ before jet starts. The vertical axis is the symmetry axis and the horizontal axis is in the equatorial plane. Slow spherical wind: at $t=0$ $v_{1}=10 \mathrm{~km} \mathrm{~s}^{-1}, \dot{M}_{1}=5 \times 10^{-5} M_{\odot} \mathrm{yr}^{-1}$ for $r<3 \times 10^{16} \mathrm{~cm}$, and $\dot{M}_{1}=10^{-5} M_{\odot} \mathrm{yr}^{-1}$ for $r>3 \times 10^{16} \mathrm{~cm}$. The jet has a half opening angle $\alpha=20^{\circ}, v_{2}=600 \mathrm{~km} \mathrm{~s}^{-1}$, and $\dot{M}_{2}=2 \times 10^{-8} M_{\odot} \mathrm{yr}^{-1}$. Arrows indicate flow direction: $v>200 \mathrm{~km} \mathrm{~s}^{-1}$ (long arrow), $20<v \leq 200 \mathrm{~km} \mathrm{~s}^{-1}$ (medium arrow), and $v \leq 20 \mathrm{~km} \mathrm{~s}^{-1}$ (short arrow). This general case can account for PNs such as NGC 6886 or NGC 3918. Bottom: Density plot at $t=530 \mathrm{yr}$ for another case. Slow spherical wind: $v_{1}=10 \mathrm{~km} \mathrm{~s}^{-1}$, $\dot{M}_{1}=5 \times 10^{-6} M_{\odot} \mathrm{yr}^{-1}$. The jet has a half opening angle $\alpha=40^{\circ}, v_{2}=600 \mathrm{~km} \mathrm{~s}^{-1}$, and $\dot{M}_{2}=2.5 \times 10^{-6} M_{\odot} \mathrm{yr}^{-1}$. This general case can account for some morphological features in He 2-104, such as the instabilities in the outer lobes. 

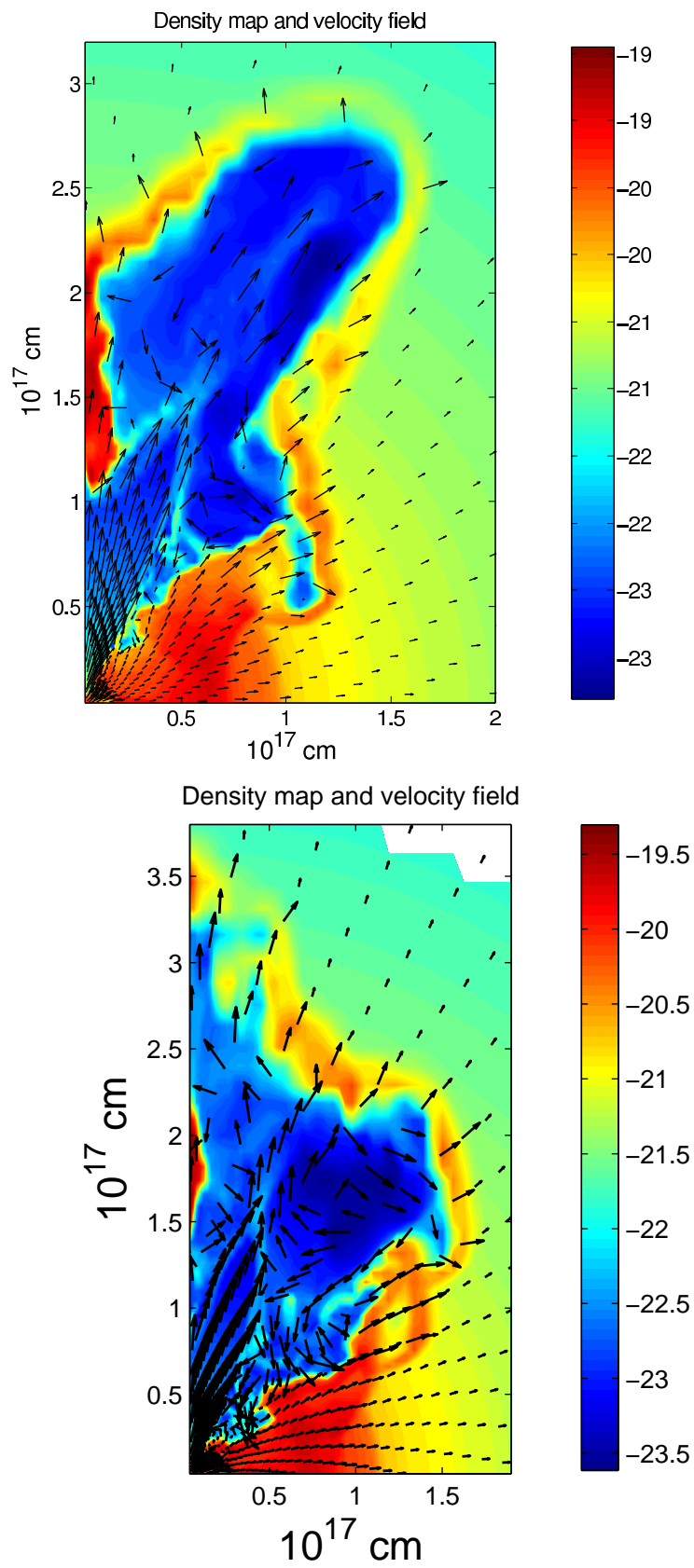

Fig. 2. Top: Density plot at $t=1300 \mathrm{yr}$ for a case with a slow wind of $v_{1}=$ $10 \mathrm{~km} \mathrm{~s}^{-1}$, and mass loss rate of $\dot{M}_{1}=3 \times 10^{-5} M_{\odot} \mathrm{yr}^{-1}$ for $r<3 \times 10^{16} \mathrm{~cm}$, and $\dot{M}_{1}=5 \times 10^{-6} M_{\odot} \mathrm{yr}^{-1}$ for $r>3 \times 10^{16} \mathrm{~cm}$ at $t=0$. The jet has a half opening angle $\alpha=30^{\circ}, v_{2}=600 \mathrm{~km} \mathrm{~s}^{-1}$, and $\dot{M}_{2}=10^{-7} M_{\odot} \mathrm{yr}^{-1}$. The initial temperature was $1000 \mathrm{~K}$, and the temperature is limited from below at $1000 \mathrm{~K}$. Bottom: Density plot at $t=1560 \mathrm{yr}$ for a case with a slow wind of $v_{1}=10 \mathrm{~km} \mathrm{~s}^{-1}$, and mass loss rate of $\dot{M}_{1}=3 \times 10^{-5} M_{\odot} \mathrm{yr}^{-1}$ for $r<3 \times 10^{16} \mathrm{~cm}$, and $\dot{M}_{1}=5 \times 10^{-6} M_{\odot} \mathrm{yr}^{-1}$ for $r>3 \times 10^{16} \mathrm{~cm}$ at $t=0$. The jet has a half opening angle $\alpha=30^{\circ}, v_{2}=600 \mathrm{~km} \mathrm{~s}^{-1}$, and $\dot{M}_{2}=10^{-7} M_{\odot} \mathrm{yr}^{-1}$. The initial temperature was $10000 \mathrm{~K}$, and the temperature is limited from below at $1000 \mathrm{~K}$. The general shape is similar to MZ 3. The arrows are just as in figure 1 Note that top and bottom are the same beside the initial temperature. 
5. In all cases that we run we saw that there is a strong dependence on the half opening angle of the jet. This will be the focus of a future paper.

\section{Acknowledgements}

I would like to acknowledge Noam Soker for his immense help with understanding the numerical simulations and for his help for getting the important results. I also thank John Blondin for his help with the numerical code. This research was supported by the Asher Space Research Institute.

\section{References}

1. Blondin J.M., 1994, The VH- 1 Users Guide, Univ. Virginia.

2. Blondin J.M., Kallman T.R., Fryxell B.A., Taam R.E., 1990, ApJ, 356, 591

3. Starrfield S., Timmes F. X., Hix W. R., Sion E. M.; Sparks W. M., Dwyer S. J., 2004, ApJ, 612, L53 